\title{
Dynamics and polarization properties of a self-modulated laser with an intracavity quarter-wave plate
}

\author{
Qian-Suo Yang *, Nai-Yi Zhu \\ Laboratory of High Temperature Gas Dynamics, Institute of Mechanics, Chinese Academy of Sciences, Beijing 100080, China
}

Received 16 March 1999; accepted 3 June 1999

\begin{abstract}
The dynamics and polarization properties of self-modulated lasers with a quarter-wave plate were numerically studied using vectorial equations. At a certain pump rate, a stable laser can operate in a self-modulation state since the insertion of a quarter-wave plate in the cavity produces a quadrature between both of the electric field components. The oscillation period is twice the cavity round-trip time and the laser field is in a swaying linear polarization state. The polarization direction rotates by $90^{\circ}$ after one cavity round-trip and, along both directions at $45^{\circ}$ and $135^{\circ}$ to the fast axis of the quarter-wave plate, an antiphase behavior is clearly demonstrated. (c) 1999 Elsevier Science B.V. All rights reserved.
\end{abstract}

Keywords: Numerical analysis; Phase shift keying; Self-pulsing lasers; Optical pulse generation; Polarization; Optical modulation; Laser amplitude stability

\section{Introduction}

Self-pulsation or self-modulation of lasers mainly results from the hysteresis in the evolution of the gain medium polarization with respect to the electric field during lasing action. If the relaxation rate of the medium polarization is much lower than the free spectral range of the laser cavity, $c / 2 L$, then the period of their self-modulation is much longer than the cavity round-trip time [1-4]. On the other hand, for a laser without any polarization-selecting elements, the instability threshold will be lower than that of the corresponding linearly polarized laser [5-7]. When the relaxation rate approaches the free spectral range, self-modulation with a frequency of the free spectral range or self-mode-locking occurs $[8,9]$.

Recent experiments have shown that self-modulation of the polarization at a frequency of half the free spectral range, $c / 4 L$, in some lasers is achievable by inserting a quarter-wave plate (QWP) into the cavity $[10,11]$. Meanwhile, the antiphase effect of the intensity components in both of the orthogonal directions has been observed. In order to explain the interesting phenomena, Brunel et al. [10] used a Jones matrix formalism for the field self-consistency after one round-trip and the beat between both of the eigenstates that oscillate in a single round-trip [10]. Obviously, the introduction of the QWP breaks the self-consistency of the vector field after one cavity round-trip, as pointed out in Refs. [12-14], and forces the polarization of the light to flip from

\footnotetext{
*Corresponding author. Fax: +86-10-6256-1284; e-mail: qsyang@imech.ac.cn
} 
TE to TM (and vice versa) each cavity round-trip. However, because this kind of laser field not only should be treated as a vector in theory but also exhibits some kind of polarization, the previous theories cannot give a complete description of this type of self-modulation, which has a high frequency of half a free spectral range. Moreover, the corresponding polarization features were not studied in the experiments mentioned above, although they have been measured in certain cases [15]. Thus, the antiphase phenomenon in this kind of laser is also not completely explained.

In this paper, using the vectorial laser equations containing the longitudinal coordinate derived in Ref. [9], the dynamics and polarization properties of a laser with a QWP in an anisotropic cavity are numerically analyzed. The insertion of the QWP forces the laser to operate in a self-modulation state with a frequency corresponding to twice the cavity round-trip. The reason for this is that the field components at $\pm 45^{\circ}$ with respect to the fast axis of the QWP rotate by $90^{\circ}$ after one cavity round-trip. Furthermore, the laser output is linearly polarized and with a swaying direction, which creates an antiphase effect of the intensity components in both of the orthogonal directions. A brief discussion of the numerical results is also given.

\section{Model description}

The typical structure of a laser with an intracavity QWP is shown in Fig. 1(a) where the angle of the fast axis with respect to the horizontal $X$ axis, i.e. the azimuth, is expressed by $\psi$ in Fig. 1(b). The losses of the field components due to the insertion of optical elements can be equivalent to the transmission losses at the end mirrors. At the left side of the gain medium, the electric field passes through the QWP backwards, is reflected by the left mirror $\mathrm{R}_{\mathrm{L}}$ and passes through the QWP in the forward direction, as shown in Fig. 1(a). This transformation of the field can be described by the following equations $(\delta=\mathrm{r}, \mathrm{i})$ :

$$
\begin{aligned}
& E_{\delta x \mathrm{f}}=E_{\delta x \mathrm{~b}} \cos 2 \psi+E_{\delta y \mathrm{~b}} \sin 2 \psi, \\
& E_{\delta y \mathrm{f}}=E_{\delta x \mathrm{~b}} \sin 2 \psi-E_{\delta y \mathrm{~b}} \cos 2 \psi,
\end{aligned}
$$

where the left mirror $\mathrm{R}_{\mathrm{L}}$ is assumed to be totally reflecting and $E_{\mathrm{r} x \mathrm{f}}, E_{\mathrm{r} y \mathrm{f}}, E_{\mathrm{i} x \mathrm{f}}, E_{\mathrm{i} y \mathrm{f}}, E_{\mathrm{r} x \mathrm{~b}}, E_{\mathrm{r} y \mathrm{~b}}, E_{\mathrm{i} x \mathrm{~b}}$ and $E_{\mathrm{i} y \mathrm{~b}}$ are the real and imaginary components of the electric fields propagating along the $Z$ and $-Z$ directions, respectively, where the subscripts $\mathrm{f}$ and $\mathrm{b}$ denote forward and backward waves, respectively.

From Ref. [9], it is known that the equations describing the dynamics and polarization properties of the vector laser field can be written as $(\delta=\mathrm{r}, \mathrm{i})$ :

$$
\begin{aligned}
& \dot{E}_{\delta x \mathrm{f}}+v \partial_{z} E_{\delta x \mathrm{f}}=g \int_{0}^{2 \pi} N(z, \theta) p_{\delta \mathrm{f}} \cos \theta \mathrm{d} \theta-\kappa E_{\delta x \mathrm{f}}, \\
& \dot{E}_{\delta y \mathrm{f}}+v \partial_{z} E_{\delta y \mathrm{f}}=g \int_{0}^{2 \pi} N(z, \theta) p_{\delta \mathrm{f}} \sin \theta \mathrm{d} \theta-\kappa E_{\delta y \mathrm{f}}, \\
& \dot{E}_{\delta x \mathrm{~b}}-v \partial_{z} E_{\delta x \mathrm{~b}}=g \int_{0}^{2 \pi} N(z, \theta) p_{\delta \mathrm{b}} \cos \theta \mathrm{d} \theta-\kappa E_{\delta x \mathrm{~b}}, \\
& \dot{E}_{\delta y \mathrm{~b}}-v \partial_{z} E_{\delta y \mathrm{~b}}=g \int_{0}^{2 \pi} N(z, \theta) p_{\delta \mathrm{b}} \sin \theta \mathrm{d} \theta-\kappa E_{\delta y \mathrm{~b}}, \\
& \dot{p}_{\delta \mathrm{f}}=g\left(E_{\delta x \mathrm{f}} \cos \theta+E_{\delta y \mathrm{f}} \sin \theta\right) d(z, \theta, t)-\gamma_{\perp} p_{\delta \mathrm{f}}, \\
& \dot{p}_{\delta \mathrm{b}}=g\left(E_{\delta x \mathrm{~b}} \cos \theta+E_{\delta y \mathrm{~b}} \sin \theta\right) d(z, \theta, t)-\gamma_{\perp} p_{\delta \mathrm{b}}, \\
& \dot{d}(z, \theta, t)=R-\gamma_{\|} \mathrm{d}(z, \theta, t)-2 g \sum_{\delta=\mathrm{r}, \mathrm{i}}\left[\left(E_{\delta x \mathrm{f}} p_{\delta \mathrm{f}}+E_{\delta x \mathrm{~b}} p_{\delta \mathrm{b}}\right) \cos \theta+\left(E_{\delta y \mathrm{f}} p_{\delta \mathrm{f}}+E_{\delta y \mathrm{~b}} p_{\delta \mathrm{b}}\right) \sin \theta\right],
\end{aligned}
$$

where $p_{\mathrm{rf}}, p_{\mathrm{if}}, p_{\mathrm{rb}}$ and $p_{\mathrm{ib}}$ are the real and imaginary parts of the polarization probability of a population inversion by the forward and backward electric fields, $d(z, \theta, t)$ is the angular density of the population inversion, the parameters $\gamma_{\|}$and $\gamma_{\perp}$ are the relaxation rates of the population inversion and medium 




(a)

(b)

Fig. 1. (a) Laser with an intracavity QWP; and (b) azimuth of the QWP in the $X-Y$ plane of the cavity.

polarization, respectively, $v$ and $\kappa$ are the propagation velocity and the losses of the electric fields, respectively, $N(z, \theta)$ and $g$, which will be normalized in the simulation process of Eq. (2), are the angular density of the active molecules and the coupling coefficient between the electric field and the gain medium, respectively, and $R$ is the pump rate.

The numerical treatment of Eq. (2) can be found in Ref. [9]. For the simplification of the simulation process, both of the parameters of the gain medium, $N(z, \theta)$ and $R$, are assumed to be isotropic in the transverse plane and uniform along the longitudinal direction. Because different kinds of gain media possess different polarization relaxation rates, $\gamma_{\perp}$, we first consider the condition $\gamma_{\perp}=2 \mathrm{~ns}^{-1}$, which corresponds to some class B lasers [16]. It will be found, by direct numerical simulation of Eq. (2), that the dynamics and polarization of the laser with a QWP under the adiabatic elimination $\gamma_{\perp} \rightarrow \infty$ are the same as those for $\gamma_{\perp}=2 \mathrm{~ns}^{-1}$. The other parameters are taken as: $L=1.8 \mathrm{~m}, \gamma_{\|}=0.0005 \mathrm{~ns}^{-1}, L_{\mathrm{m}}=40 \mathrm{~mm}, R_{\mathrm{R} x}=0.95, R_{\mathrm{R} y}=0.93$ and $R_{\mathrm{L} x}=\mathrm{R}_{\mathrm{L} y}$ $=1$. The laser is divided into 180 sections and there is a loss of $0.002 \mathrm{~ns}^{-1}$ in each section occupied by the gain medium and no loss for the sections without the gain medium. Furthermore, the gain medium is assumed to be close to the left mirror $\mathrm{R}_{\mathrm{L}}$.

\section{Numerical results and discussion}

Fig. 2(a) shows both of the intensity components of the electric field forward at the right mirror in the $X$ and $Y$ directions in the stable region and their peak values in the unstable region as a function of the pump rate $R$ without a QWP. In the stable region, the electric field is linearly polarized along the $X$ direction because of a higher reflectivity of the left mirror to the field component in this direction and the intensity is directly proportional to the pump rate $R$. In the unstable region, where the pump rate is above the self-modulation threshold $R_{\mathrm{sm}}$, the maximum of both of the intensities is also directly proportional to the pump rate $R$, although self-modulation with a frequency of the free spectral range appears. After a QWP is inserted into the cavity at an azimuth of $\psi=45^{\circ}$, as shown in Fig. 2(b), the laser threshold, which is also the self-modulation threshold, is higher than the former laser threshold but lower than the self-modulation threshold $R_{\mathrm{sm}}$. Comparison of our numerical analysis with the experimental results of Loh et al. [11] shows that the lack of a stable region in our numerical result may be attributed to the fact that spontaneous emission effects are not involved in the laser equations. In fact, the modulation of the electric field due to the insertion of QWP can be covered by the spontaneous emission since the light intensity is small near the laser threshold $R_{\mathrm{th}}$. However, similar to that observed in the experiment of Loh et al. [11], the relationship between the pump rate and the light intensity in the unstable region in our numerical results, as shown in Fig. 2(b), is linear.

In the self-modulation state of the laser without a QWP, oscillation in the $Y$ direction with a lower reflectivity at the right mirror has a minimum value for the light intensity of zero, as shown in Fig. 3. In fact, the self-oscillation of the field component in the $X$ direction also exists but with a small amplitude on top of a high 

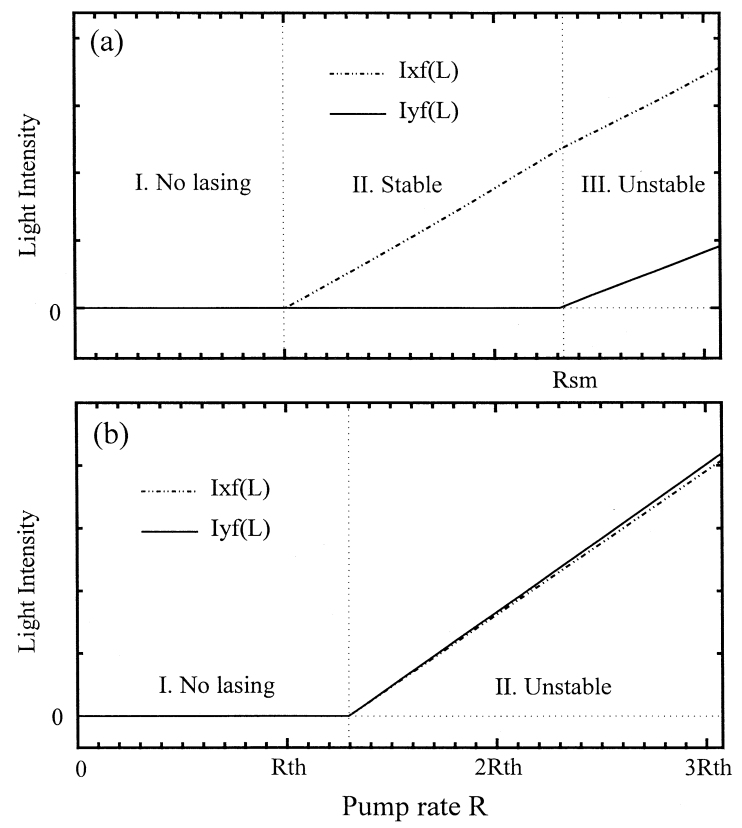

Fig. 2. The two forward intensity components at the right mirror, $I_{x \mathrm{f}}(\mathrm{L})$ and $I_{y \mathrm{f}}(\mathrm{L})$, in the stable state and their peak values in the unstable state as a function of pump rate $R$ : (a) without QWP where $R_{\mathrm{th}}=6.5 \times 10^{-5} \mathrm{~ns}^{-1}$ is the laser threshold and $R_{\mathrm{sm}}$ is the self modulation threshold; and (b) with a QWP of azimuth $\psi=45^{\circ}$.


Fig. 3. (a) Waveforms of the forward electric field components at the right mirror; and (b) waveforms of the corresponding intensities in an unstable state without any QWP where the pump rate $R$ in each section is taken as $2.77 R_{\mathrm{th}}>R_{\mathrm{sm}}$. 
dc level, which can be seen in Fig. 3(a) and Fig. 3(b). The simulation also reveals that the electric field is linearly polarized and with a swaying direction, i.e. the four components satisfy the following equation $(\eta=\mathrm{f}, \mathrm{b})$ [15]:

$$
E_{\mathrm{r} x \eta}=E_{\mathrm{i} x \eta}, E_{\mathrm{r} y \eta}=E_{\mathrm{i} y \eta}, E_{\mathrm{r} x \eta} E_{\mathrm{i} y \eta}-E_{\mathrm{r} y \eta} E_{\mathrm{i} x \eta}=0 .
$$

Furthermore, the components in the $X$ and $Y$ directions oscillate with a frequency of the free spectral range, as shown in Fig. 3(a) and Fig. 3(b).

The insertion of a QWP at $\psi=45^{\circ}$ between the left mirror $\mathrm{R}_{\mathrm{L}}$ and the gain medium can change the stable output of this laser into the self-modulation state. Eq. (1) is used to describe the process of the electric field passing through the QWP. Fig. 4 shows the component waveforms of the electric field, the intensity and the corresponding phase portraits. It is obvious that the oscillation frequencies of the laser field equals half the free spectral range, $c / 4 L$. Meanwhile, the component waveforms of the electric field and the intensity in the $X$ and $Y$ directions display an antiphase behavior, as shown in Fig. 4, which is the same as in the previous experimental results $[10,11]$. This phenomenon can be explained by the linear polarization, with a periodically swaying direction, of the laser field where the real and imaginary parts of the $x$ and $y$ electric field components are identical in the numerical solution of Eq. (2). Because the electric field components pass through the zero point, the minimum of the corresponding intensity components is the zero point, as shown in Fig. 4.

The self-modulation of the laser with a QWP as a function of the azimuth angle $\psi$ is also investigated for a fixed pump rate, at which the laser output is stable if there is no QWP in the cavity. The maximum and minimum of the $x, y$ intensity components versus $\psi$ are shown in Fig. 5(a). It is easy to see that the modulation depth of the laser field gradually increases as $\psi$ varies from 0 to $45^{\circ}$, where the cases of $\psi=0^{\circ}$ and $\psi=45^{\circ}$ correspond to the stable state and the complete modulation state, respectively. The minimum of the intensity in the $X$ direction decreases from the fixed value of the $\mathrm{CW}$ state to the zero point. The maximum in the $Y$


Fig. 4. Waveforms of: (a) the forward electric field components at the right mirror; and (b) their intensities; where $\left(\mathrm{a}^{\prime}\right)$ and $\left(\mathrm{b}^{\prime}\right)$ show the corresponding phase portraits for the laser with a QWP of azimuth $\psi=45^{\circ}$ and the pump rate $R$ is taken as $2.153 R_{\mathrm{th}}<R_{\mathrm{sm}}$. 

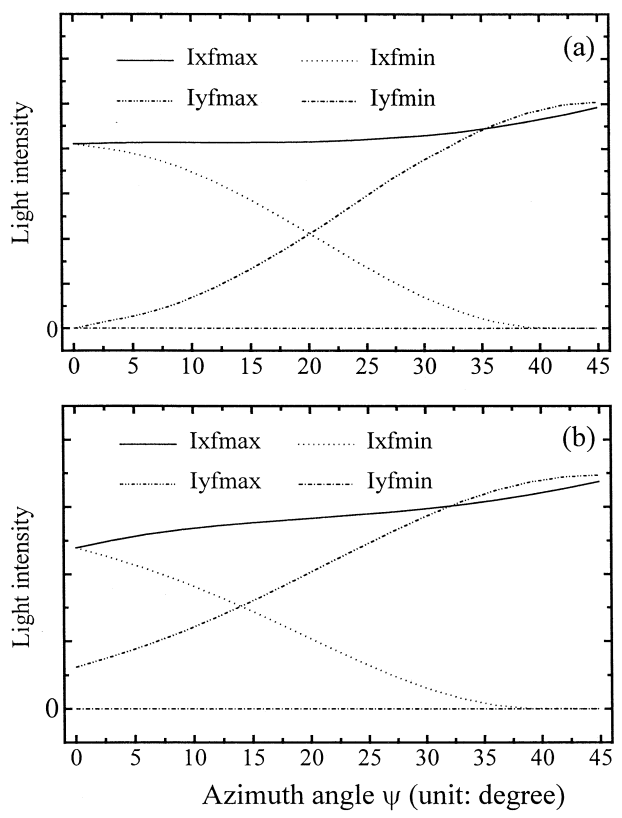

Fig. 5. Intensity components, $I_{x \mathrm{f} \max }, I_{x \mathrm{f} \min }, I_{y \mathrm{f} \max }$, and $I_{y \mathrm{f} \text { min }}$, at the right mirror versus the QWP's azimuth $\psi$ : (a) in the stable state with $R=2.153 R_{\mathrm{th}}$; and (b) in the unstable state with $R=2.77 R_{\mathrm{th}}$.

direction increases from the zero point at the stable state while the minimum stays at the zero point. It is also found that, although the QWP can be placed in the cavity with different azimuths, the modulation frequency always equals half the free spectral range, the state of linear polarization with a swaying direction is constant and the antiphase effect of the $x, y$ intensity components does not disappear. With a pump rate above the self-modulation threshold $R_{\mathrm{sm}}$, the diagram of the maximum of the intensity component in the $Y$ direction versus the azimuth of the QWP increases from non-zero intensity, as shown in Fig. 5(b). Fig. 6 shows the component waveforms of the electric field and the intensity where the pump rate $R$ and the QWP's azimuth $\psi$ are taken as $2.153 R_{\mathrm{th}}$ and $30^{\circ}$, respectively. From this, one can find that the self-modulation of the field component in the $X$ direction is also on top of a dc level.

Under the adiabatic elimination of $\gamma_{\perp} \rightarrow \infty$, Eq. (2) can be written as $(\delta=\mathrm{r}, \mathrm{i})$ :

$$
\begin{aligned}
& \dot{E}_{\delta x \mathrm{f}}+v \partial_{z} E_{\delta x \mathrm{f}}=\frac{g^{2} N(z, \theta)}{\gamma_{\perp}} \int_{0}^{2 \pi}\left(E_{\delta x \mathrm{f}} \cos \theta+E_{\delta y \mathrm{f}} \sin \theta\right) \cos \theta d(z, \theta, t) \mathrm{d} \theta-\kappa E_{\delta x \mathrm{f}}, \\
& \dot{E}_{\delta y \mathrm{f}}+v \partial_{z} E_{\delta y \mathrm{f}}=\frac{g^{2} N(z, \theta)}{\gamma_{\perp}} \int_{0}^{2 \pi}\left(E_{\delta x \mathrm{f}} \cos \theta+E_{\delta y \mathrm{f}} \sin \theta\right) \sin \theta d(z, \theta, t) \mathrm{d} \theta-\kappa E_{\delta y \mathrm{f}}, \\
& \dot{E}_{\delta x \mathrm{~b}}-v \partial_{z} E_{\delta x \mathrm{~b}}=\frac{g^{2} N(z, \theta)}{\gamma_{\perp}} \int_{0}^{2 \pi}\left(E_{\delta x \mathrm{~b}} \cos \theta+E_{\delta y \mathrm{~b}} \sin \theta\right) \cos \theta d(z, \theta, t) \mathrm{d} \theta-\kappa E_{\delta x \mathrm{~b}}, \\
& \dot{E}_{\delta y \mathrm{~b}}-v \partial_{z} E_{\delta y \mathrm{~b}}=\frac{g^{2} N(z, \theta)}{\gamma_{\perp}} \int_{0}^{2 \pi}\left(E_{\delta x \mathrm{~b}} \cos \theta+E_{\delta y \mathrm{~b}} \sin \theta\right) \cos \theta d(z, \theta, t) \mathrm{d} \theta-\kappa E_{\delta y \mathrm{~b}} \\
& \dot{d}(z, \theta, t)=R-\gamma_{\|} d(z, \theta, t)-2 \frac{g^{2}}{\gamma_{\perp}} \sum_{\delta=\mathrm{r}, \mathrm{i}}\left[\left(E_{\delta x \mathrm{f}} \cos \theta+E_{\delta y \mathrm{f}} \sin \theta\right)^{2}+\left(E_{\delta x \mathrm{~b}} \cos \theta+E_{\delta y \mathrm{~b}} \sin \theta\right)^{2}\right] \\
& \quad \times d(z, \theta, t) .
\end{aligned}
$$



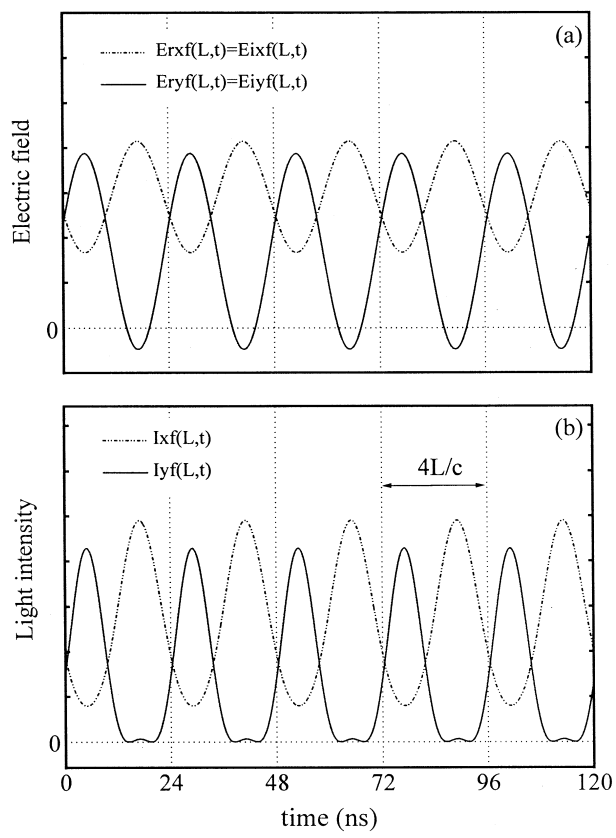

Fig. 6. (a) Waveforms of the forward electric field components at the right mirror; and (b) the corresponding intensities where the QWP's azimuth $\psi$ and the pump rate $R$ are taken as $30^{\circ}$ and $2.153 R_{\text {th }}$, respectively.

Under the normalization of $N(z, \theta), g$ and $\gamma_{\perp}$ and with the same values for the other parameters, we also obtain that self-modulation of the laser with a QWP of azimuth $\psi=45^{\circ}$ exists near the threshold. Although the waveforms of the fields are different from those above, the modulation period still equals twice the cavity round-trip time, the linearly polarized laser field still swings with a period of $4 L / c$ and, therefore, the antiphase effect of both orthogonal directions can be observed.

The self-modulation of lasers with a QWP can be explained as follows. From Eq. (1), it can be seen that the components of the electric field in the direction having an angle of $45^{\circ}$ with respect to the fast or slow axis will rotate towards the orthogonal direction after one round-trip in the cavity and return to the same direction after two cavity round-trips. Meanwhile, using Eq. (1), we obtain:

$$
\begin{aligned}
E_{\mathrm{r} x \mathrm{f}} E_{\mathrm{i} y \mathrm{f}}-E_{\mathrm{r} y \mathrm{f}} E_{\mathrm{i} x \mathrm{f}} & =\left(E_{\mathrm{r} x \mathrm{~b}} \cos 2 \psi+E_{\mathrm{r} y \mathrm{~b}} \sin 2 \psi\right)\left(E_{\mathrm{i} x \mathrm{~b}} \sin 2 \psi-E_{\mathrm{i} y \mathrm{~b}} \cos 2 \psi\right) \\
& -\left(E_{\mathrm{r} x \mathrm{~b}} \sin 2 \psi-E_{\mathrm{r} y \mathrm{~b}} \cos 2 \psi\right)\left(E_{\mathrm{i} x \mathrm{~b}} \cos 2 \psi+E_{\mathrm{i} y \mathrm{~b}} \sin 2 \psi\right) \\
& =E_{\mathrm{r} y \mathrm{~b}} E_{\mathrm{i} x \mathrm{~b}}-E_{\mathrm{r} x \mathrm{~b}} E_{\mathrm{i} y \mathrm{~b}}=0 .
\end{aligned}
$$

Therefore, the linear polarization feature of the laser field does not change after it passes through the QWP twice. Because the electric field can be expressed into the superposition of the components in both of the directions having an angle of $45^{\circ}$ and $-45^{\circ}$ with respect to the fast axis and the polarization of both components is self-consistent after two cavity round-trips, the modulation frequency is half the free spectral range. On the other hand, when both field components with the same amplitude are amplified by the gain medium, a difference in net gain difference breaks their equality after one cavity round-trip. Furthermore, the rotation of the field polarization caused by the QWP, during the next cavity round-trip time, induces the effect that the strongest component in a given direction will be amplified by the gain-distribution having the lowest gain-saturation and, therefore, the highest gain in this direction, and vice versa. This directional consistency of the strongest component of the electric field and the highest gain results in the self-modulation of the linear 
polarized field. Meanwhile, the antiphase phenomenon arises from the periodic sway of the polarization direction.

\section{Conclusion}

In summary, with the vectorial laser equations, the dynamics and polarization property of self-modulated laser with an intracavity QWP are numerically investigated. The insertion of the QWP makes the vector field achieve self-consistency after two cavity round-trips, which leads to the polarization of the light within the gain medium having a preferential orientation in the direction of the dominant gain as pointed out in Ref. [9], and, therefore, forces the laser to pulsate. Thus, the corresponding modulation frequency is half the free spectral range. Moreover, the laser field is linearly polarized but with a swaying polarization direction. The periodic alternation of the polarization direction causes an antiphase effect in both of the orthogonal directions.

\section{Acknowledgements}

The authors acknowledge helpful discussions with Dr. Peng-Ye Wang and Prof. Ling-An Wu.

\section{References}

[1] M. Maeda, N.B. Abraham, Measurements of mode-splitting self-pulsing in a single-mode, Fabry-Perot laser, Phys. Rev. A 26 (6) (1982) 3395-3403.

[2] R.S. Gioggia, N.B. Abraham, Routes to chaotic output from a single-mode, dc-excited laser, Phys. Rev. Lett. 51 (8) (1983) $650-653$.

[3] C.O. Weiss, J. Brock, Evidence for Lorenz-type chaos in a laser, Phys. Rev. Lett. 57 (22) (1986) 2804-2806.

[4] H. Haken, Light Volume 2 Laser Light Dynamics, North-Holland, 1985, pp. 187-214.

[5] G.P. Puccioni, M.V. Tratnik, J.E. Sipe, G.L. Oppo, Low instability threshold in a laser operating in both states of polarization, Opt. Lett. 12 (4) (1987) 242-244.

[6] N.B. Abraham, M.D. Matlin, R.S. Gioggia, Polarization stability and dynamics in a model for a polarization-isotropic laser that goes beyond third-order Lamb theory, Phys. Rev. A 53 (5) (1996) 3514-3528.

[7] E. Lacot, F. Stoeckel, M. Chenevier, Dynamics of an erbium-doped fiber laser, Phys. Rev. A 49 (5) (1997) $3997-4008$.

[8] H. Risken, K. Nummedal, Self-pulsing in laser, J. Appl. Phys. 39 (10) (1968) 4662-4672.

[9] Q.-S. Yang, P.-Y. Wang, J.-H. Dai, H.-J. Zhang, Vectorial equations and polarization characteristics of pulsed lasers, IEEE J. Quantum Electron. 34 (1998) 1135-1141.

[10] M. Brunel, M. Vallet, G. Ropars, A. Le Floch, F. Bretenaker, Model analysis of polarization self-modulated lasers, Phys. Rev. A 55 (2) (1997) 1391-1397.

[11] W.H. Loh, Y. Ozeki, C.L. Tang, High-frequency polarization self-modulation and chaotic phenomena in external cavity semiconductor lasers, Appl. Phys. Lett. 56 (26) (1990) 2613-2615.

[12] W.H. Loh, C.L. Tang, Numerical investigation of ultrahigh frequency polarization self-modulation in semiconductor lasers, IEEE J. Quantum Electron. 27 (1991) 389.

[13] C.L. Tang, Laser polarization self-modulation effects, Quantum Semiclass. Opt. 10 (1) (1998) R51-R58.

[14] W.H. Loh, C.L. Tang, Successive higher-harmonic bifurcation of a polarization self-modulated external cavity semiconductor laser, Opt. Commun. 85 (1991) 283-290.

[15] Q.-S. Yang, P.-Y. Wang, J.-H. Dai, H.-J. Zhang, Alternating linear polarization state in a neodymium-doped fiber laser with modulated pump, Chin. Phys. Lett. 14 (11) (1997) 834-837.

[16] C.O. Weiss, R. Vilaseca, Dynamics of Lasers, VCH, Weinheim, 1991, pp. 213-214. 